\title{
Enhancing Cloud Scaling by Reducing Slow Data Migrations using Supervised Learning Approach
}

\author{
Dr. R. Poorvadevi ${ }^{* 1}$, L.Laxmi narayana Reddy ${ }^{2}$, G.V. Suhas Reddy ${ }^{3}$ \\ ${ }^{1}$ Assistant Professor, CSE Department, SCSVMV University, Kanchipuram. \\ ${ }^{2 \& 3}$ UG Student, CSE Department, SCSVMV University, Kanchipuram
}

\begin{abstract}
In the current era of computing world, the entire web based services and application are processed in the different cloud service vendors regions. The application/service specific processes are executed with the proper SLA segment which brings the access control over cloud users. When the user's application, services and resources are migrated from one network region to another region, the migration time factor is notified in the cloud service provider region. It helps to users and service provider to check how the services are migrated with the proper time span in the specific service access environment. The slow level data migrations are identified and the service regions may check the occurrences of slow migration and the cloud scaling need to be enhanced in the cloud service environment. The proposed work will be used to make the decisions towards eliminating the slow data migrations and increase the legibility level of cloud scaling factor.
\end{abstract}

Keywords - Service Level Agreement; Cloud Service Provider; Cloud Vendor; Cloud Scaling; Cloud Users; Controller Area Network; Data Migration; Service Optimization

\section{Introduction}

In cloud computing, the distinct user services are processed in the various cloud service provider locations. The functional processes are executed with the specific access level constraints and examining the level of service trustworthiness is monitored to check the level of service optimization among the user community. When the user wants to relocate their services in the other service region then, cloud data migration comes in the picture. When clients are migrating the applications and other resource kind of data, users need to verifying which controller are network (CAN) user wants to migrate the services. The various set of applications optimized and the level of cloud service scaling is determined with the proper virtual machine monitor. Because virtual machine executes all internal processes are executed with access level constraints to check the service integrity. The VMM needs to verify the access location and type of services from the concern users region. The cloud scaling is needed to be enhanced in the cloud service access regions to ensure the level of service optimization.

\section{Literature Survey}

As per an auhor Fei Zhang, Guangming Liu et.al, "A survey on virtual machine migration: challenges, techniques and open issues". This paper stated that, how the virtual machine migration are processed in the service access region and what are the major challenges are faced during the time of service deployment and migration moment. This work has been resulted into the finding the limitations and techniques in the cloud service access platform with the respect to the mechanism of virtual machine migration strategy. [1]

An author Michael Menzel, Rajiv Ranjan et.al, "CloudGenius: A hybrid decision support method for automating the migration of web application clusters to public clouds". This paper focused on the hybrid decision support method for automating the process of web service based applications and the cluster user groups are identified. When clients are accessing the services in public cloud model all migration strategies are deployed with the specific cloud vendor region. [2]

Author Quanwang Wu, Fuyuki Ishikawa et.al, "Energy and migration Cost-Aware Dynamic Virtual Machine Consolidation in Heterogeneous cloud Datacenters". This approach was implemented on the heterogeneous platform and evaluated the migration cost aware dynamic virtual machine. It was mainly focused on the consolidation of distinct VM's in the various cloud datacenter regions. [3]

\section{Proposed Work}

In cloud computing environment, the application specific processes are running in the different virtual machine. When the user services, data and resources are migrated from one VM to another VM then cloud service provider need to examine the network delay, transmission speed, data accuracy and other service based constraints. Migration is one of the major process in cloud computing. It leads the user to distribute the services to another user in the datacenter location. When transferring the users 
application from one cloud access vendor to other region user may face the problem slow data migration. It needs to be solved in the concern workflow where users are suffering with the cloud scalability factor.

.......The following components are used in the proposed work for enhancing the level of cloud scalability which is listed below:

- User service provisioning

- Access cloud region

- SLA ID

- Type of service need to be migrated

- Service operated segment ID

- Migration time

- Network service process

- Cloud region process

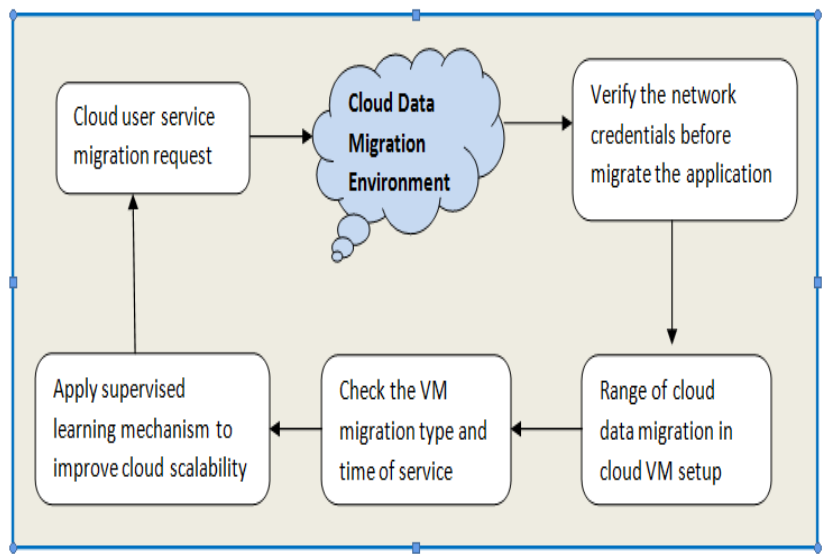

Fig.1: Illustration of Proposed System Workflow Model

An above diagram depicts the functional processes of how the migration is carried out and executed in the cloud vendor region and also providing the platform to identify the slow data migrations in the user specific environment. The various components are considered in the cloud migration system to find and avoid the slow level data migration which is listed below:

- Specify End-user devices (Smartphone, tablet, laptop, desktop)

- Network (yours and/or cloud provider's)

- Cloud services (IaaS, PaaS, SaaS)

- Infrastructure (Server/VM)

- Web server

- APIs

- App itself

Above said components were adopted in the public cloud deployment model and the migration process are analyzed with the cloud indicator. It needs to be ensuring the application performance to measure the different key metrics and end-end workflow has been processed in the cloud model.

\section{Implementation}

All the application specific performance processes are enabled with the kind of application what user id preferring and monitoring performances are majorly include the following parameters.

- Crashes

- Errors

- Service Performance Response Time

- Network

- Battery

- Signal strength

It is important to ensure internal applications before deploying in to the app store location. The third party apps need many application performance monitoring vendors which provides the mobile apps to be refined properly. The various steps are used to find the slow migration cloud services / data/ resources are to be built in the cloud environment.

- Separate problems to the code, network, or infrastructure layers, and use stack trace at code-level to speed resolution.

- Analyze the app across the devices and OS versions, and carriers to identify trends.

- Track usages, crashes, errors, performance of HTTP, and volume relative to thresholds and geography.

- Evaluate the recital of mobile apps across geographies, carriers, devices, and OS to optimize performance.

- Trace transactions from the user, network, and into the backend.

- Reconstruct incidents to fix issues across datacenters, cloud services, and containers or micro services.

\subsection{Importance of Supervised Learning in Proposed Model}

The main aim of supervised learning will be used to develop predictive model based on both input and output data for any application which needs to be migrated in the different cloud vendor locations. This approach will discover the relationship between input attributes and a target attribute. It is represented in the structured model of application process.

Classification process includes the type of data or services migrated, vendor location, specify the occurrences of client access level information, detailed process of migration timing and network properties and also examine the capacity of finding slow data migration in VM access location. Regression involve in the process of finding the input variables. Environment from where the migration initiated in the user location, network error, access privileges verification and other constraints are also determined in this process.

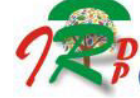




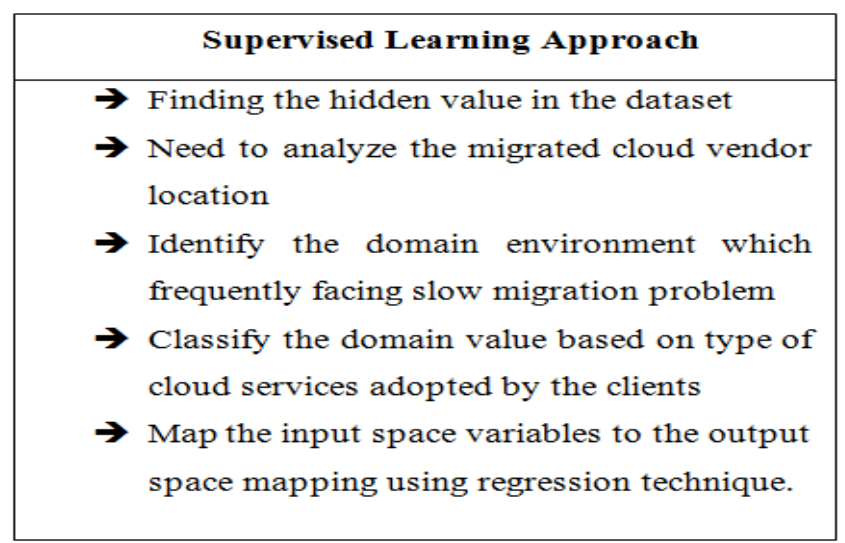

Fig: 2 Process of Supervised Learning Approach

\section{Experimental Results}

The following table drive the functional process outcome of slow data migration which facilitates the indication of processing the client data access with the migration policies which needs to be indicated in the cloud user service access environment. It needs to be refined in the public cloud service model type and specific application and service parameters are calculated and identified.

Table 1: The rate of slow data migration in public cloud

\begin{tabular}{|l|l|l|l|}
\hline $\begin{array}{l}\text { Service ID in Cloud } \\
\text { platform }\end{array}$ & $\begin{array}{l}\text { Device Level } \\
\text { process status }\end{array}$ & $\begin{array}{l}\text { Type of Issue in the } \\
\text { Migration Process }\end{array}$ & $\begin{array}{l}\text { Indicating } \\
\text { Process status }\end{array}$ \\
\hline Region-1a-34.9/e & Migrated & Attack identified & Determined \\
\hline Region Group-11s/9.67-1 & Migrated & Network error caused & Processed \\
\hline $\begin{array}{l}\text { Region Access zone - } \\
\text { 39.86/L }\end{array}$ & Optimized & IP spoofed data & Iterated \\
\hline $\begin{array}{l}\text { Region block segment ID: } \\
\text { 189.83/bs/cloud vendor 3 }\end{array}$ & Processed & Enabled data source & Established \\
\hline
\end{tabular}

An above table indicates the process view of reducing the slow data migration occurrences and finding the correct cloud platform to monitor the process of data migration. This table process in the different cloud region group and examines the process segment of each cloud user application will be examined in the general cloud process in the specific cloud region.

Figure 3 shows the experimental outcomes of slow data migration process in the cloud region. The various service access platform need to be monitored and service based network issues also monitored. The process statuses are indicated in the cloud service provider and user region and the process control need to be updated.

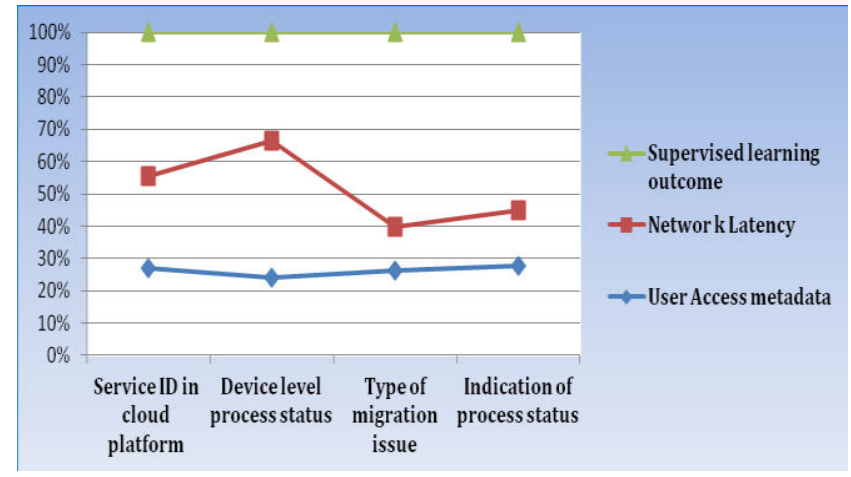

Fig.3: Illustration of Slow Data Migration Process Outcome

\section{Conclusion}

In cloud user specific application programs all the client level processes are monitored and the various service access level constraints are verified with the proper privileges and the cloud scaling need to be enhanced in the client level process by reducing the slow data migration problem. It helps to increase the factor of cloud service agility process over the cloud service access platform.

\section{References}

[1] Fei Zhang ; Guangming Liu ; Xiaoming Fu ; Ramin Yahyapour, "A Survey on Virtual Machine Migration: Challenges, Techniques, and Open Issues", IEEE Journal on Communications Surveys \& Tutorials, Volume: 20, Issue: 2, Year: 2018

[2] Michael Menzel; Rajiv Ranjan; Lizhe Wang; Samee U. Khan ; Jinjun Chen, "CloudGenius: A Hybrid Decision Support Method for Automating the Migration of Web Application Clusters to Public Clouds", Journal on IEEE Transactions on Computers, Volume: 6,Year: 2015.

[3] Quanwang Wu; Fuyuki Ishikawa; Qingsheng Zhu; Yunni Xia, "Energy and Migration Cost-Aware Dynamic Virtual Machine Consolidation in Heterogeneous Cloud Datacenters", IEEE Transactions on Services Computing, Volume: 12, Issue: 4, Year 2019.

[4] Ruitao Xie ; Yonggang Wen ; Xiaohua Jia ; Haiyong Xie, "Supporting Seamless Virtual Machine Migration via Named Data Networking in Cloud Data Center", IEEE Transactions on Parallel and Distributed Systems, Volume: 26, Issue: 12 ,Year: 2015

[5] Yaodong Yang; Bo Mao; Hong Jiang; Yuekun Yang; Hao Luo; Suzhen Wu, "SnapMig: Accelerating VM Live Storage Migration by Leveraging the Existing VM Snapshots in the Cloud", IEEE Transactions on Parallel and Distributed Systems, Volume: 29, Issue: 6,Year: 2018

[6] Bo Hu ; Shanzhi Chen ; Jianye Chen ; Zhangfeng Hu, "A MobilityOriented Scheme for Virtual Machine Migration in Cloud Data Center Network", IEEE journal on cloud computing, Volume: 4, Year: 2016.

[7] Yang Wang; Wei Shi ; Menglan Hu, "Virtual Servers CoMigration for Mobile Accesses: Online versus Off-Line", IEEE Transactions on Mobile Computing, Volume: 14, Issue: 12, Year: 2015. 